\section{Por que reconsiderar a indicação do rastreamento do câncer de mama?}

\author{
Why reconsider the recommendation of breast \\ cancer screening?
}

¿Por qué reconsiderar la indicación del rastreo del cáncer de mama?
Charles Dalcanale Tesser 1

Thiago Luiz de Campos d'Ávila 1,2

\title{
Resumo
}

O objetivo deste artigo foi discutir a recomendação do rastreamento mamográfico do câncer de mama e suas bases técnicas. A primeira parte tece considerações sobre critérios de decisão, que devem ser coerentes com evidências científicas de alta qualidade. A segunda discute o sobrediagnóstico, o maior dano do rastreamento, e seu significado questionador do modelo da história natural da doença. A terceira parte sintetiza estudos sobre eficácia, efetividade e danos do rastreamento, mostrando que os últimos (principalmente sobrediagnósticos e falsos positivos) são significativos e tornam a balança entre danos e benefícios duvidosa. Conclui-se que a recomendação de rastreamento mamográfico deve ser reconsiderada pelas autoridades sanitárias brasileiras em qualquer idade.

Programas de Rastreamento; Neoplasias da Mama; Prevenção Secundária 


\section{Introdução}

O Ministério da Saúde brasileiro recomenda o rastreamento de câncer de mama para mulheres entre 50 e 69 anos por meio de mamografia bienal 1, e manteve isto na recente consulta pública sobre o tema 2. Mas não existe no país um programa organizado de rastreamento de base populacional, que implicaria o convite sistemático, acompanhamento das rastreadas, garantia de diagnóstico e tratamento e avaliação do processo. Os programas organizados têm maior impacto sobre a mortalidade atribuída à condição rastreada e são menos onerosos que os rastreamentos oportunísticos (quando a pessoa procura um profissional e é realizado o rastreamento) 3 .

$\mathrm{O}$ rastreamento mamográfico sempre suscitou controvérsias, mais quanto à idade para seu início. Nos Estados Unidos, após anteriores indicações de rastrear também aos 40-49 anos, em 2009 a recomendação foi restrita para acima de 50 anos, bienalmente, o que está hoje em processo de revisão ${ }^{4}$. As recomendações europeias, em que a maior parte dos programas organizados ocorre, também indicam o início aos 50 anos 5,6. Como a idade é o maior fator de risco, mulheres mais velhas se beneficiam mais com menos danos, pois realizam menos exames do que as mais jovens 7. Dado que o objetivo maior é reduzir a mortalidade 8,9,10, faz sentido que o rastreamento não seja indicado entre 40-49 anos, sendo esta a recomendação da Bélgica, Dinamarca, Finlândia, Itália, Polônia, Holanda e Reino Unido 11.

Recentemente, estudos idôneos, como os da Colaboração Cochrane, têm questionado a recomendação de rastreamento mamográfico $12,13,14,15,16,17$. A mudança deve-se provavelmente ao sobrediagnóstico, que, junto com os demais danos, associou-se à pequena eficácia do rastreamento em ensaios clínicos e à sua pouca (ou nula) efetividade na morbimortalidade de populações reais, fazendo a avaliação danos x benefícios ficar duvidosa. Uma instituição médica nacional europeia recentemente sugeriu a interrupção desse rastreamento 18 .

A visão crítica da indicação do rastreamento suscita reações contrárias 19. Outros complicantes são a dificuldade de mensuração do sobrediagnóstico e a valorização do seu significado, dado não haver para ele uma explicação frente ao modelo da história natural da doença utilizado. Isso requer discussão a respeito dos fundamentos das decisões sobre a indicação dos rastreamentos.

O objetivo deste artigo é discutir a recomendação do rastreamento mamográfico do câncer de mama a partir de suas principais bases técnicas. Apresentam-se aspectos técnicos envol- vidos no estabelecimento das indicações dos rastreamentos, articulando elementos conceituais e técnicos com resultados empíricos que subsidiam a questão. Tal discussão envolve as recomendações governamentais, com influência direta sobre as práticas dos profissionais do Sistema Único de Saúde (SUS) (as quais não são aqui abordadas).

Metodologicamente, não se trata de uma revisão sistemática da literatura. Antes, trata-se de um "ensaio", mas não somente teórico: uma discussão envolvendo critérios, saberes e evidências dirigidas à decisão sobre a recomendação. O primeiro tópico considera os critérios técnicos para o estabelecimento da recomendação do rastreamento. Neles, estão embutidos aspectos éticos, como em toda intervenção médica e de saúde pública, abordados apenas o necessário para compreensão do processo de decisão. Uma abordagem teórica, ética ou bioética não caberia neste espaço. O segundo discute o sobrediagnóstico, com apontamentos sobre a adequação do modelo da história natural da doença e do diagnóstico anatomopatológico usado como padrão-ouro de interpretação comum aos casos diagnosticados por sintomas clínicos e aos rastreados. O terceiro tópico sintetiza estudos sobre aspectos da eficácia, efetividade e danos, que são a base empírica da conceituação do sobrediagnóstico e da reavaliação da recomendação deste rastreamento.

\section{Rastreamento: aspectos básicos, conceitos, decisões}

Os requisitos técnicos dos programas organizados de rastreamento são muito diferentes das situações em que há adoecimento, sintomas e sofrimento, com relação à tolerância aos danos, à garantia de benefícios e ao manejo da incerteza. No caso do cuidado ao adoecimento sentido, o sofrimento presente autoriza razoável grau de intervenção diagnóstica e terapêutica com grande margem de incerteza, em função da expectativa de benefício da terapêutica. Não se cobra resultado favorável, mas a correção técnica e ética da ação profissional.

No caso do rastreamento, a relação com a tolerância aos danos, a garantia de benefícios e o manejo da incerteza são radicalmente diferentes. Há que ser imensamente mais conservador e precavido para com as intervenções, tanto diagnósticas quanto terapêuticas ${ }^{20}$. Como os rastreamentos são testes realizados em indivíduos assintomáticos, as exigências para a sua indicação são altíssimas, pois os danos iatrogênicos não estão contrabalançados com a compensação da 
cura, controle ou alívio do adoecimento sentido. Os benefícios estão ancorados em um potencial de evitação de adoecimento e morte futuros para um grupo pequeno de indivíduos (os beneficiados), dentro de um universo bem maior de sadios (todos expostos ao potencial de danos). Nesse caso, o princípio da não-maleficência (não causar danos) impera sobre o da beneficência (desejo de promover o bem-estar), visto que pessoas que se percebem como saudáveis podem ter sua saúde abalada indefinidamente devido à intervenção ${ }^{21}$. Nessa situação, é imperativo ter a certeza de que os benefícios são amplamente maiores do que os riscos e danos 3,22.

Ocorrem danos devido ao fato de pessoas assintomáticas, com o rastreamento, serem tratadas como "quase" doentes, com implicações psicológicas e sociais: ansiedade e incertezas dos falsos positivos, falsa segurança dos falsos negativos e situações limítrofes que requerem monitoramento continuado, por exemplo. Apesar das evidências serem maiores para os efeitos psicológicos da mamografia, sobretudo de ansiedade e a curto prazo ${ }^{23}$, muitas pessoas passam a se sentir doentes pela vivência dos atendimentos e exames 24 , e impactos psicológicos podem persistir por anos após um câncer ser descartado 25 . Isso abala as capacidades culturais e individuais para lidar com a doença, a dor e a morte; e instala medos previamente inexistentes 26. Mesmo informadas com noções sobre risco, no rastreamento as pessoas podem receber "uma gota de tinta na água cristalina de suas identidades, que poderá não mais ser clareada" 27 (p. 222).

Há danos e desconfortos provocados diretamente pelos procedimentos diagnósticos, as sequelas físicas de complicações resultantes dos exames e tratamentos e, no caso do câncer de mama, principalmente, há o sobrediagnóstico. “Todos os rastreamentos causam danos e alguns fazem bem" 28 (p. 292). Por isso, a indicação de um rastreamento em um programa organizado ou fora dele pelas autoridades sanitárias demanda necessariamente segurança e garantia de resultados.

A decisão deve ser estritamente coerente com e dependente do melhor, mais sólido e atualizado saber científico disponível, visando à garantia de benefícios sem danos ou com mínimos deles. Um cientificismo (decisões estritamente baseadas em evidências científicas) nos rastreamentos é reforçado pela argumentação esboçada por Rose 29 , dividindo as ações preventivas em dois tipos. As chamadas ações de prevenção "a menos" ou redutiva são de correção de excessos ou faltas, erros alimentares ou desvios no modo de viver sabidamente patogênicos: parar de fumar, diminuir sedentarismo, ingesta alcoólica excessiva, diminuir alimentos processados. Elas são teoricamente coerentes e corroboradas pelos conhecimentos científicos disponíveis, havendo consenso relativamente bem estabelecido sobre isto, com progressiva importância da promoção da saúde em dimensão societal e individual. Trata-se do restabelecimento de modos de viver salutogênicos, não problemáticos quanto à fundamentação da sua recomendação 29 .

O segundo tipo são as ações de prevenção "a mais" ou aditiva: intervenções heterônomas (administradas profissionalmente) estranhas à ecologia usual da pessoa, com aplicação de fármacos, testes diagnósticos, vacinas ou outros produtos biológicos, físicos ou químicos. Como há maior potencial de dano e não há adoecimento sentido (a priori as pessoas são saudáveis), a intervenção pode resultar em danos sem potenciais benefícios 28 , como indicam as caselas 3 e 5 da Tabela 1, ilustrativo dos desfechos do rastreamento.

A postura, nesse caso, deve ser oposta: do consenso afirmativo da prevenção redutiva, deve-se passar ao ceticismo negativista e criterioso na prevenção aditiva. O ônus da prova deve recair nos propositores da intervenção. A garantia de benefícios e de não-maleficiência deve existir e recai inteiramente nos profissionais e sistemas de saúde 30: deve haver evidências científicas substanciais e consensuais de que os benefícios superam os danos (que devem ser nulos ou mínimos).

A situação permite uma analogia com o direito: o réu (cidadão) é inocente até que se prove o contrário e, em dúvida, pró réu. As pessoas são saudáveis e assim ficarão até que seu adoecimento (ou um rastreamento) prove o contrário: um

Tabela 1

Situações possíveis nos rastreamentos.

\begin{tabular}{|c|c|c|}
\hline & $\begin{array}{l}\text { Doente clinicamente no } \\
\text { futuro }\end{array}$ & $\begin{array}{c}\text { Não-doente clinicamente } \\
\text { no futuro }\end{array}$ \\
\hline Rastreamento negativo & $\begin{array}{l}\text { 1. Falsos negativos } \\
\text { (falsa segurança) }\end{array}$ & 2. Verdadeiros negativos \\
\hline $\begin{array}{l}\text { Rastreamento falso } \\
\text { positivo }\end{array}$ & - & $\begin{array}{l}\text { 3. Falsos positivos * } \\
\text { (danos) }\end{array}$ \\
\hline $\begin{array}{l}\text { Rastreamento positivo } \\
\text { confirmado }\end{array}$ & $\begin{array}{l}\text { 4. Verdadeiros positivos * } \\
\text { (benefícios) }\end{array}$ & $\begin{array}{l}\text { 5. Sobrediagnósticos * } \\
\text { (danos) }\end{array}$ \\
\hline
\end{tabular}

* Podem haver danos além dos específicos da situação (derivados dos sobrediagnósticos e dos falsos positivos, nas respectivas caselas), como dores, hematomas e hemorragias quando há biopsia etc.

Fonte: inspirado em esquema de Gray 28. 
resultado positivo confirmado será considerado prova, mas a confirmação tem margem de erro e este processo todo pode causar dano. Em casos de balanço desfavorável entre danos e benefícios ou dúvida nessa avaliação, pelo princípio da precaução, do primum non nocere e por prudência não deve ser recomendado o rastreamento (como ilustrado na Figura 1).

Na dúvida, deve-se assumir a inocência ("saudabilidade”) dos réus (assintomáticos) e não se recomenda rastreamento; procura-se melhorar diagnose e tratamento dos sintomáticos, orientar prevenção redutiva e promover a saúde. Há forte assimetria entre recomendação e não-recomendação. Se há necessidade do balanço ser amplamente favorável aos benefícios para haver recomendação positiva, apenas a dúvida deve ser suficiente para sustentar a não-recomendação.

As complexidades na avaliação das evidências e do balanço danos x benefícios são muitas e isto gera gradações nas recomendações, favoráveis e desfavoráveis. Do ponto de vista do significado prático, quando há evidências consensuais de que o balanço é favorável, recomenda-se o rastreamento (situação 1 da Figura 1). As situações 2, 3 e 4 da Figura 1 têm em comum um sig-

Figura 1

Proposta de significados das recomendações de rastreamento.

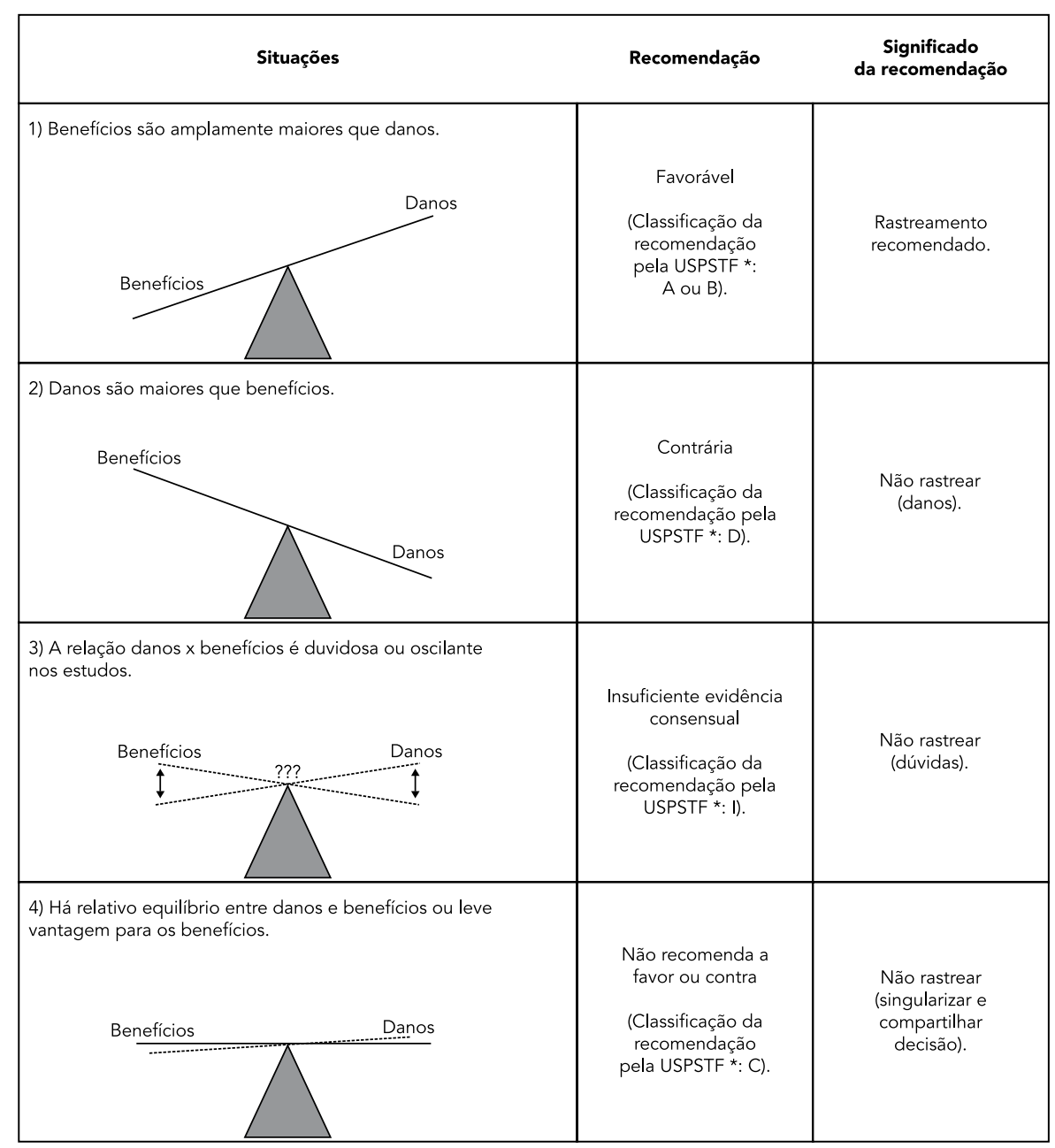

* USTFPF: U.S. Preventive Services Task Force, cuja classificação das recomendações é amplamente usada no mundo e no Brasil 3,4. 
nificado de não-recomendação. Essa varia de recomendação contra (2); passa por uma não-recomendação em situação duvidosa (3), conforme antes discutido; e desdobra-se, no caso de "equilíbrio" entre danos e benefícios ou fraca vantagem dos benefícios (4), em não-recomendação, geralmente traduzida como indicando decisão compartilhada e singularizada entre usuário e profissional. Nesse último caso, renuncia-se à normatividade da recomendação e aproxima-se do anterior, tendendo a uma não-recomendação, dado o ceticismo conservador (resistente à ação) desejável na prevenção aditiva com danos significativos, como é o caso. A decisão pela implantação/manutenção de um programa organizado ou pela emissão de recomendação favorável de rastreamento demanda claramente a situação 1 da Figura 1.

A garantia de segurança e de balanço favorável só pode ser obtida por meio da avaliação de estudos epidemiológicos de alta qualidade: revisões e metanálises de ensaios clínicos aleatorizados que mensurem benefícios e danos e, implantado o programa, avaliação da sua efetividade, focando redução populacional da mortalidade geral e específica e incidência nos vários estágios da doença 8,9 .

Os resultados de tais estudos podem ser (e amiúde são) anti-intuitivos e estão fora do alcance da experiência clínica dos profissionais. Por isso, sua opinião não deve ter posição privilegiada. Diferentemente do cuidado ao adoecimento sentido, em que pequena parte dos processos decisórios é suprida por evidências de boa qualidade, as decisões nos rastreamentos devem ser baseadas estritamente nas melhoras e mais atualizadas evidências, porque elas são a única fonte confiável de informação. Isso é reforçado pela complexidade dos processos de saúde-doença, pela ignorância sobre a gênese e evolução dos cânceres e pelas dificuldades de avaliação dos efeitos (com seus vieses de tempo de antecipação, de duração e o sobrediagnóstico) e dos danos dos rastreamentos 9,20,30,31,32,33,34. A decisão requer estudos em escala coletiva e populacional de eficácia e efetividade envolvendo também avaliação dos danos, principalmente do sobrediagnóstico, este completamente invisível à experiência de doentes e médicos nas situações clínicas.

\section{Entendendo o sobrediagnóstico}

O sobrediagnóstico é conceituado como a situação em que a pessoa é diagnosticada com uma condição que jamais lhe causaria sintomas ou morte. Ocorre quando o diagnóstico é realizado corretamente, em alguém sem sintomas relacionados à condição diagnosticada. Diferentemente do diagnóstico falso positivo (detectado em um exame e posteriormente descartado), no sobrediagnóstico do câncer o indivíduo tem, de fato, uma alteração histológica que preenche os critérios diagnósticos 12,35.

No rastreamento do câncer de mama o sobrediagnóstico não é um fenômeno manifesto; antes, é uma hipótese para explicar o descompasso entre o aumento sustentado da incidência de cânceres após o início dos rastreamentos, desproporcional à pouca ou nenhuma alteração da mortalidade (em relação a populações ou grupos não rastreados) e da morbidade (não redução proporcional das formas avançadas de câncer). Se há aumento sustentado dos diagnósticos totais (incidência) à custa dos estágios iniciais sem redução proporcional da mortalidade e/ou das formas avançadas, a hipótese que resta é que estes novos diagnósticos pós-rastreamento não eram de doenças que levariam à morte, como se esperava, pois se o fossem a incidência das formas avançadas e a mortalidade iriam necessariamente diminuir.

O sobrediagnóstico é uma explicação adequada aos achados empíricos tanto dos melhores ensaios clínicos quanto dos estudos comparativos das mortalidades e incidências nas populações rastreadas e não rastreadas, como sintetizado no próximo tópico. Ele questiona implicitamente o padrão-ouro diagnóstico dos cânceres e o modelo da história natural da doença, usados conjuntamente para dar sentido e fundamento ao rastreamento e ao tratamento precoce pré-sintomático. Uma clara definição da doença e sua evolução são o primeiro passo para uma proposta de rastreamento, uma vez que o conhecimento bem estabelecido sobre a evolução da mesma em fase pré-sintomática dever ser consensualmente aceito ${ }^{34}$.

Os rastreamentos foram desenvolvidos dentro do modelo da história natural das doenças 36 , que induz à crença de que as doenças progredirão ao longo do tempo, a não ser que se intervenha 21 . Essa ideia, apesar de amplamente utilizada nas pesquisas e no cotidiano dos médicos, é questionável em muitos casos. A "história natural" é um conceito relativo que não deveria generalizar-se para além de algumas enfermidades infecciosas, pois foi desenvolvido no século XIX em torno das mesmas. Sua realidade é duvidosa inclusive em muitos processos infecciosos (gripe, leishmaniose, tuberculose, dengue ou lepra) e também no lupus eritematoso, esquizofrenia, diabetes, hérnia de disco lombar, demência, depressão e câncer de mama ${ }^{37}$. São cada vez mais numerosos os exemplos de doenças, anormali- 
dades e disfunções diagnosticáveis por métodos sofisticados progressivamente mais sensíveis, e com pouca ou nenhuma correlação com sintomas clínicos ou desfechos mórbidos. Autores têm chamado a esse fenômeno de reservatório de doenças 38 , ou pseudo-doenças 39 .

A história natural do câncer de mama é relativizada pela emergência do sobrediagnóstico, que poderia ter uma de duas explicações. O câncer sobrediagnosticado nunca progride e pode, inclusive, entrar em remissão; ou progride lentamente e o indivíduo morre por outras causas antes de ter desenvolvido qualquer sintoma do câncer. O segundo caso envolveria três variáveis: o tamanho do câncer quando detectado, sua taxa de crescimento e a expectativa de vida do novo paciente. Mesmo um câncer de crescimento rápido pode gerar um sobrediagnóstico, quando for muito pequeno em tamanho e/ou ocorrer em uma pessoa com limitada expectativa de vida 39 .

Visando manter a adequação empírica, relativizando a "história natural" da doença e o anatomopatológico como padrão-ouro diagnóstico, e reconhecendo a ignorância trazida à luz pelos achados indicativos do sobrediagnóstico, introduziu-se uma hipótese para esclarecer parcialmente a situação: a ideia de que há diferenças entre o câncer detectado pelo rastreamento (câncer "histológico") e o diagnosticado pelos sintomas (câncer "biológico"), visto que podem ser situações de naturezas distintas e apresentarem evoluções e desfechos diferentes 33 . O primeiro pode ficar latente ou remitir, ou avançar para o segundo, mas uma parte dos casos não o faz, permanecendo como um estoque de alterações que não se manifesta clinicamente. Isso permite entender o sobrediagnóstico como a detecção desse estoque, que gera incidência aumentada (diagnóstico e tratamento), mas não reduz proporcionalmente a mortalidade nem diminui a incidência de formas graves.

Para que o sobrediagnóstico ocorra é necessário haver um reservatório substancial de anormalidades histologicamente iguais ao câncer distribuído na população, parte do qual não terá consequência clínica. Tal reservatório é composto por numerosas alterações histológicas subclínicas, assintomáticas, porém detectáveis. Atividades que levem à detecção do reservatório são pré-requisitos para a ocorrência do sobrediagnóstico, sendo o rastreamento de câncer a principal delas 38 .

A diferença entre câncer histológico e biológico necessita explicação. Uma tentativa de estudar o problema seria mudar a nomenclatura diagnóstica dos casos diagnosticados por rastreamento. Esserman et al. 35 propõem, dentre outras estratégias, que o termo "câncer" deveria ser reservado para descrever lesões com uma probabilidade razoável de progressão fatal se não tratado. A diferenciação das denominações permitiria distinguir o grupo diagnosticado via rastreamento do diagnosticado por sintomas clínicos, acompanhá-los e desenhar investigações objetivando esclarecer quais fatores ou processos estão envolvidos na transformação ou não do câncer histológico em biológico. Isso possibilitaria estudos epidemiológicos sobre a evolução desses cânceres e minimizaria danos decorrentes do uso do termo câncer para os diagnósticos derivados do rastreamento.

Hoje, um câncer detectado pelo rastreamento mamográfico é enquadrado em três categorias: um câncer clinicamente importante que tem maiores chances de ser curado se descoberto antes (o benefício da mamografia); um câncer também clinicamente importante, mas que não será mais tratável, mesmo descoberto precocemente (muito agressivo); ou um câncer sobrediagnosticado, que não causará sintomas ou morte. Até $90 \%$ dos cânceres detectados em rastreamentos mamográficos estão nas duas últimas categorias 12 .

Apesar de ser possível, analisando retrospectivamente programas de rastreamento, identificar e estimar o sobrediagnóstico, o maior problema é reconhecer as sobrediagnosticadas 38 . O sobrediagnóstico na pessoa só pode ser identificado se ela recusar o tratamento, viver livre de sintomas e morrer por outras causas; o que praticamente não ocorre. Todos os diagnosticados são tratados 12 e para quem é sobrediagnosticado não há benefício no sobretratamento, apenas danos. As evidências mais fortes do sobrediagnóstico provêm de estudos sobre rastreamentos do câncer de mama 40 .

\section{O sobrediagnóstico, danos e benefícios no rastreamento mamográfico}

Acreditava-se que a mamografia permitiria que as mulheres evitassem tratamentos mais agressivos ao realizar o diagnóstico precoce e detectar tumores menores e mais fáceis de tratar. Estudos mostraram, todavia, que a mamografia tem esse efeito nulo 41,42 ou ao contrário: aumento de $20 \%$ de mastectomias realizadas 12,15 .

O sobrediagnóstico é o dano mais importante dos rastreamentos. Sua introdução em vários países está associada com o aumento considerável de novos casos de câncer de mama. Espera-se um aumento inicial de casos com o início dos programas (lesão assintomática precocemente detectada), porém, com o tempo, esse aumento seria inibido ao se reduzir o número de casos 
detectados tardiamente ${ }^{12}$. No entanto, a análise de Bleyer \& Welch 43 da incidência de câncer de mama em três décadas de rastreamento nos Estados Unidos mostra que a incidência de câncer em estágio inicial aumentou drasticamente, e a incidência de câncer em estágio final diminuiu de maneira insignificante, sugerindo duas conclusões: primeiro, que o rastreamento é falho ao não adiantar o tempo de diagnóstico dos cânceres causadores de morte. Segundo, que o rastreamento está relacionado a um número expressivo de sobrediagnósticos. Mesmo ao excluir-se o excesso na incidência associado com a terapia de reposição hormonal, estima-se que nos Estados Unidos cerca de 1,3 milhão de mulheres foram sobrediagnosticadas 44 .

A magnitude do sobrediagnóstico varia nos estudos: $10 \% 45,22 \% 46,31 \% 43$ até $52 \% 47$ (se forem considerados os carcinoma in situ dos diagnósticos por rastreamento). Duas revisões recentes envolvendo populações grandes em países europeus, Estados Unidos, Canadá e Austrália, concluíram que a taxa de sobrediagnóstico é de $30 \% 14,15$. A cada 2 mil mulheres rastreadas por 10 anos, apenas uma terá sua vida salva pelo rastreamento; 10 serão sobrediagnosticadas e sobretratadas; e 200 experimentarão importante estresse psicológico incluindo ansiedade e incerteza devido a falsos positivos 14,15. Em outra revisão 47 , de cada 10 mil mulheres rastreadas por 20 anos, aproximadamente 43 evitariam a morte por câncer de mama; cerca de 129 seriam tratadas desnecessariamente devido ao sobrediagnóstico: três sobrediagnosticadas para cada morte por câncer evitada. A maioria das mulheres com câncer detectado pelo rastreamento não terá suas vidas salvas, mas será diagnosticada antes sem alteração na mortalidade ou sobrediagnosticada 14,48 . A metáfora jurídica novamente ilumina a situação: não se aceita, em geral, que para identificar/ punir um criminoso, inocentes sejam lesados na investigação/pena. Mas algo semelhante ocorre no sobrediagnóstico: vários inocentes (pessoas saudáveis) são lesados (sobretratados) para que um criminoso (câncer clinicamente significativo no futuro) seja punido (tratado). Mas como não são identificáveis os inocentes lesados (confundidos com criminosos), eles não reclamam, não exigem reparos.

Quanto aos falsos positivos, estes dependem da qualidade das mamografias, são abundantes e aumentam quanto menor a idade. Uma revisão Cochrane 15 estima entre 20 e $60 \%$ a chance acumulada de um falso positivo em 10 mamografias de rastreamento, e Elmore et al. 49 a calculam em $49,1 \%$.

Há outros danos, como maior mortalidade devido à doença cardíaca, bem como indução de câncer de pulmão nas mulheres rastreadas 15 . Ocorrem outros efeitos adversos e complicações dos procedimentos, menos estudados. Biópsias podem produzir complicações (perfuração de artéria, infecção, alergias). Estima-se que metade das mulheres sobrediagnosticadas sofrerá com dor crônica pelo sobretratamento 15 .

Quanto aos benefícios, a redução de mortalidade geral é o desfecho ideal de avaliação, mas alguns estudos mostraram não haver esta redução ${ }^{15,48}$. Na falta desse critério, resta a redução da mortalidade por câncer de mama em ensaios clínicos. Essa varia de 046 a $20 \% 50,51$, sendo a estimativa mais mencionada entre 10 e $15 \% 15$ (porém, entre os estudos aleatorizados de maneira adequada, os melhores não apresentaram diferença na mortalidade 15). Aceitando a redução de $20 \%$ no risco relativo, isso significa uma redução de risco absoluto de apenas $0,1 \%$ (a mortalidade por câncer de mama reduz de 5 para 4 mulheres por mil nas rastreadas). Bleyer \& Welch 43 questionam se essa pequena diminuição da mortalidade nas populações rastreadas deve-se à mamografia. Argumentam que a mortalidade caiu devido aos melhores tratamentos e à descoberta de novos quimioterápicos, e mostram que o grupo que teve a diminuição da mortalidade foi o de mulheres com doença regional (com linfonodo positivo). Essas mulheres foram as mais beneficiadas pelo tratamento, mesmo entre as que não foram rastreadas e descobriam o câncer por sintomas. A taxa de mortalidade no grupo com doença metastizada permaneceu inalterada ao longo das três décadas avaliadas, com ou sem rastreamento. Outra recente revisão sistemática de estudos de sete países também concluiu que a taxa de câncer em estágio avançado não foi alterada pelo rastreamento 52 . Além disso, o declínio na mortalidade das mulheres expostas ao rastreamento é o mesmo verificado nas mulheres abaixo dos 40 anos que não foram rastreadas 43 . Logo, parece que a pequena redução na mortalidade tem como importante corresponsável melhorias no tratamento e não apenas o rastreamento. Isso foi reforçado pelo mais longo estudo comparando mortalidades de populações reais rastreadas e não rastreadas (35 anos analisados, 20 pré e 15 pós-introdução do rastreamento), que não mostrou redução da mortalidade nas rastreadas comparadas às não rastreadas 53 . Por último, um recente ensaio clínico canadense acompanhou mulheres rastreadas anualmente e não rastreadas (que receberam exame médico rotineiro anual) entre 40 e 49 anos por 25 anos, e mostrou não haver redução de mortalidade no grupo rastreado 46 .

Além disso, enquanto os benefícios do rastreamento de câncer de mama parecem ter sido 
inicialmente superestimados e hoje mensurados como pequenos, supondo existirem, os danos comumente são omitidos: aproximadamente apenas um terço dos ensaios clínicos quantifica os danos do rastreamento 54 e somente $13 \%$ dos artigos sobre o rastreamento mamográfico citam o sobrediagnóstico como possibilidade, havendo ainda uma diferença grande entre periódicos de medicina geral e de especialidades focais. Esses últimos são mais explícitos em rejeitar dados de revisões sistemáticas e apresentam, com maior frequência, problemas de conflitos de interesses 55 .

Dimensões culturais e atores sociais complexificam os danos. A difusão na mídia e em campanhas dos benefícios e a omissão/minimização dos danos induzem as mulheres a realizarem o rastreamento acreditando ser apenas benéfico. Gera-se uma tendência de ressignificação do processo de envelhecer das mulheres, levandoas a acreditar que é perigoso não rastrear. Por intermédio de uma divulgação que incita o medo, gera-se uma pressão pelo rastreamento. Uma antiga propaganda da Sociedade Americana de Câncer dizia que se uma mulher não quer fazer a mamografia, ela precisa examinar outra coisa além das mama 12. Também os médicos mencionam mais os benefícios do que os danos nas conversas com suas pacientes 56 .

Há ainda o chamado paradoxo da popularidade 9 , um processo importante na disseminação da pressão por mamografia, de origem técnica, uma vez que gerado pelo sobrediagnóstico: todas as mulheres sobrediagnosticadas acreditam terem sido beneficiadas. Apesar de prejudicadas, acreditam que foram "salvas" pelo rastreamento e assim o popularizam. Elas difundem e defendem o rastreamento como uma necessidade, utilizando seu exemplo pessoal como alerta e exemplo, em um contexto em que cada vez mais as mulheres conhecem alguém que teve câncer de mama.

O sobrediagnóstico gera outro efeito psicossocial danoso: um "falso risco" familiar. A cascata iniciada pelo sobrediagnóstico repercute na geração subsequente, pois filhas e irmãs de mulheres com câncer de mama comumente se sentem com maior risco.

Alguns atores sociais e institucionais brasileiros reforçam a pressão pelo rastreamento, podendo isto ser interpretado como iatrogenia cultural 57. A campanha "outubro rosa" tinge de rosa cidades e instituições, divulgando o rastreamento mamográfico 58,59. Também instituições especializadas fazem o mesmo ${ }^{60,61}$. Quase não discutem os danos e recomendam às mulheres: "faça sua mamografia anual a partir dos 40 anos de idade" 60.
Esse conjunto complexo de dados e situações torna o rastreamento mamográfico no mínimo duvidoso 15,16. Por isso, estaria indicado, ao rastrear as mulheres individualmente, colher um consentimento informado, após discussão clara de riscos e benefícios 31. Alguns programas de rastreamento estão revendo o conteúdo de suas cartas-convite, incluindo informação sobre falsos positivos e sobrediagnóstico (por exemplo, a Inglaterra) 59 .

Um limite da discussão precedente é que pode padecer de viés de seleção. Somente várias revisões sistemáticas poderiam esgotar os múltiplos aspectos envolvidos. Esforço nesse sentido foi visível na recente consulta pública do governo brasileiro sobre o tema ${ }^{2}$, cujos dados convergem com os aqui reunidos. Embora uma mudança evidente de postura tenha ocorrido, expressa na afirmação de que a ênfase em atingir ampla cobertura do rastreamento mamográfico no Brasil deve ser substituída pela prioridade em garantir os possíveis benefícios e diminuir os malefícios, a conclusão do documento oficial continuou a ser favorável ao rastreamento, sem argumentação substancial quanto ao balanço em questão. A introdução da dúvida parece estar indicada no texto governamental, mas, se significou inequívoca mudança de perspectiva, todavia não refletiu integralmente nas recomendações, que essencialmente se mantiveram positivas quanto ao ponto principal.

\section{Considerações finais}

O pequeno benefício de redução da mortalidade - aceitando existir - é fortemente contrabalançado por significativos danos nas mulheres rastreadas: mensuráveis (abundantes falsos positivos, aproximadamente $30 \% 40$ de sobrediagnósticos e suas consequências, biópsias desnecessárias, complicações associadas) e imensuráveis (visitas médicas adicionais, mudanças psicológicas e sociais, medos antes inexistentes, percepção aumentada vitalícia de risco de câncer para gerações subsequentes, situações limítrofes com longo follow-up, paradoxo da popularidade). O balanço entre danos e benefícios é no mínimo duvidoso 16,62.

Todavia, revisões continuam recomendando o rastreamento, como em Pace \& Keating 63, e a Organização Mundial da Saúde (OMS) 64 recentemente manteve a recomendação, mas apenas em locais com bons recursos e/ou com sistema de saúde forte, que sustente um programa organizado de rastreamento de base populacional, em que a participante seja bem informada dos benefícios e riscos. Dois argumentos principais aparecem 
sustentando essa posição favorável condicional. Primeiro, a diversidade das estimativas da magnitude do sobrediagnóstico tornaria essa evidência fraca 64 . Segundo, a OMS adotou a posição de duas outras revisões de 2012 em que a proporção de um ou dois casos de sobrediagnóstico para cada morte evitada foi considerada aceitável, um "balanço apropriado" 64. Essa argumentação é frágil, se não precária: apenas assumir a adequação do balanço mencionado é questionável em si mesmo; e mais questionável ainda se for considerado que outros estudos apontam proporções de 347 até 1015 casos sobrediagnosticados para cada morte evitada. A OMS também desconsiderou que os estudos com melhor qualidade apontam para menos benefícios 14,15 e maior proporção de sobrediagnóstico 14,15, o que reforça a evidência e o significado deste último.

A magnitude e natureza dos danos, sobretudo o sobrediagnóstico, frente à pequenez dos benefícios (se existirem), considerados os critérios discutidos para a decisão sobre rastreamentos, introduz no mínimo a dúvida, o que autorizaria postura contrária à recomendação positiva do rastreamento ${ }^{62,65}$. Por outro lado, o Brasil não é um local com bons recursos ou que sustente um programa de rastreamento organizado ${ }^{2}$. Essas ponderações sugerem a reconsideração da recomendação do rastreamento mamográfico pelas autoridades sanitárias brasileiras.

\section{Colaboradores}

C. D. Tesser participou de todas as etapas da elaboração do artigo (concepção, redação inicial, revisões e redação final, e atualização bibliográfica). T. Luiz Campos participou da redação de partes significativas (tópicos segundo e terceiro) do artigo, e análise e discussão da literatura envolvida.

\section{Agradecimentos}

Agradecemos a Armando Henrique Norman pela discussão de conteúdos do artigo e ao CNPq pelo subsídio financeiro (processo no 312989/2013-0) e pela bolsa de pós-doutoramento (processo no 207603/2014-6). 


\section{Referências}

1. Instituto Nacional de Câncer José Alencar Gomes da Silva. Controle do câncer de mama - Documento de Consenso. http://www.inca.gov.br/pu blicacoes/consensointegra.pdf (acessado em 12/ Jan/2015).

2. Secretaria de Atenção à Saúde, Ministério da Saúde. Diretrizes para a detecção precoce do câncer de mama no Brasil. http://www2.inca.gov.br/ wps/wcm/connect/31e01e8045c5a619bacefe dad9df0b60/\%27201410091145_Diretrizes_Ma ma_Brasil_10_2014.pdf?MOD=AJPERES\&CACHEI

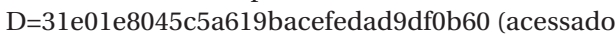
em 23/Jun/2015).

3. Departamento de Atenção Básica, Secretaria de Atenção à Saúde, Ministério da Saúde. Rastreamento. Brasília: Ministério da Saúde; 2010. (Série A. Normas e Manuais Técnicos) (Caderno de Atenção Primária, 29).

4. U.S. Preventive Services Task Force. Screening for breast cancer: U.S. Preventive Services Task Force recommendation statement. Ann Intern Med 2009; 151:716-26.

5. International Agency for Research on Cancer. IARC Working Group on the Evaluation of CancerPreventive Strategies. Breast cancer screening. Lyon: International Agency for Research on Cancer; 2002. http://www.iarc.fr/en/publications/pdfsonline/prev/handbook7/Handbook7_Breast.pdf (acessado em 18/Abr/2016).

6. Altobelli E, Lattanzi A. Breast cancer in European Union: an update of screening programmes as of March 2014 (Review). Int J Oncol 2014; 45:1785-92.

7. U.S. Preventive Services Task Force. Effects of mammography screening under different screening schedules: model estimates of potential benefits and harm. Ann Intern Med 2009; 151:738-47.

8. Rosser W, Shafir M. Evidence-based family medicine. Hamilton: BC Decker Inc.; 1998.

9. Raffle A, Gray M. Screening evidence and practice. Oxford: Oxford University Press; 2007.

10. Sackett DL, Haynes RB, Guyatt GH, Tugwell P. Early diagnosis. In: Sackett DL, Haynes RB, Guyatt GH, Tugwell P, editors. Clinical epidemiology: a basic science for clinical medicine. 2nd Ed. Boston: Lippincott Williams and Wilkins; 1991. p. 153-70.

11. Giordano L, von Karsa L, Tomatis M, Majek O, de Wolf C, Lancucki L, et al. Mammographic screening programmes in Europe: organization, coverage and participation. J Med Screen 2012; 19 Suppl 1:72-82.

12. Welch HG. Overdiagnosed: making people sick in the pursuit of health. Boston: Beacon Press; 2011

13. Gøtzsche PC. Time to stop mammography screening? CMAJ 2011; 183:1957-8.

14. Gøtzsche PC, Nielsen M. Screening for breast cancer with mammography. Cochrane Database Syst Rev 2011; (1):CD001877.

15. Gøtzsche PC, Jørgensen KJ. Screening for breast cancer with mammography. Cochrane Database Syst Rev 2013; (6):CD001877.

16. Elmore JG, Kramer BS. Breast cancer screening - toward informed decisions. JAMA 2014; 311: 1298-9.
17. Kalager M, Adami HO, Bretthauer M. Too much mammography. Long term follow-up does not support screening women under 60. BMJ 2014; 348:g1403.

18. Swiss Medical Board. Systematic mammography scrennig. http://www.medical-board.ch/filead min/docs/public/mb/Fachberichte/2013-12-15_ Bericht_Mammographie_Final_Kurzfassung_e.pdf (acessado em 18/Abr/2016).

19. Biller-Andorno N, Jüni P. Abolishing mammography screening programs? A view from the Swiss Medical Board. N Engl J Med 2014; 370:1965-7.

20. Norman AH, Tesser CD. Rastreamento de doenças. In: Gusso G, Lopes JMC, organizadores. Tratado de medicina de família e comunidade: princípios, formação e prática. Porto Alegre: Editora Artmed; 2012. p. 521-32.

21. Norman AH. Ética médica e rastreamento: em quais evidências deveríamos nos apoiar? Rev Bras Med Fam Comunidade 2014; 9:105-7.

22. Instituto Nacional de Câncer. Controle do câncer de mama. Documento de consenso. Rio de Janeiro: Instituto Nacional de Câncer; 2004.

23. Brett J, Bankhead C, Henderson B, Watson E, Austoker J. The psychological impact of mammographic screening. A systematic review. Psychooncology 2005; 14:917-38.

24. Kloetzel K. Medicina ambulatorial: princípios básicos. São Paulo: Editora EPU; 1999.

25. Brewer NT, Salz T, Lillie SE. Systematic review: the long-term effects of false-positive mammograms. Ann Intern Med 2007; 146:502-10.

26. Gérvas J, Starfield B, Heath I. Is clinical prevention better than cure? Lancet 2008; 372:1997-9.

27. Sweeney K. Science, society, suffering and the self: a commentary on general practice for the twenty first century. New Zealand Family Practice 2005; 32:221-4.

28. Gray JAM. New concepts in screening. Br J Gen Pract 2004; 54:292-8.

29. Rose G. Estratégias da medicina preventiva. Porto Alegre: Editora Artmed; 2010.

30. Gates TJ. Screening for cancer: evaluating the evidence. Am Fam Physician 2001; 63:513-22.

31. Marshall KG. Prevention. How much harm? How much benefit? 4 . The ethics of informed consent for preventive screening programs. Can Med Assoc J 1996; 155:377-82.

32. Gates TJ. Cancer screening in perspective. Am Fam Physician 2001; 63:1039-43.

33. Zahl PH, Maehlen J, Welch HG. The natural history of invasive breast cancers detected by screening mammography. Arch Intern Med 2008; 168:2311-6.

34. Wilson JMG, Jungner G. Principles and practice of screening for disease. Geneva: World Health Organization; 1968. (Public Health Papers, 34).

35. Esserman L, Shieh Y, Thompson I. Rethinking screening for breast cancer and prostate cancer. JAMA 2009; 302:1685-91.

36. Leavell H, Clark EG. Medicina preventiva. São Paulo: McGrawHill do Brasil; 1976. 
37. Pérez-Fernández M, Gérvas J. El efecto cascada implicaciones clínicas, epidemiológicas y éticas. Med Clin (Barc) 2002; 118:65-7.

38. Welch GH. Should I be tested for cancer? Maybe not and here's why. Berkeley: University of California Press; 2004.

39. Welch HG, Black WC. Overdiagnosis in cancer. J Natl Cancer Inst 2010; 102:605-13.

40. Moynihan R, Doust J, Henry D. Preventing overdiagnosis: how to stop harming the healthy. BMJ 2012; 344:e3502.

41. Surhke P, Mæhlen J, Schlichting E, Jørgensen KJ, Gøtzsche PC, Zahl PH. Mammography screening and surgical breast cancer treatment in Norway: comparative analysis of cancer registry data. BMJ 2011; 343:d4692.

42. Nederend J, Duijm LEM, Voogd AC, Groenewoud $\mathrm{JH}$, Jansen FH, Louwman MWJ. Trends in incidence and detection of advanced breast cancer at biennial screening mammography in The Netherlands: a population based study. Breast Cancer Res 2012; 14:R10.

43. Bleyer A, Welch HG. Effect of three decades of screening mammography on breast-cancer incidence. N Engl J Med 2012; 367:1998-2005.

44. Zackrisson S, Andersson I, Janzon L, Manjer J, Garne JP. Rate of over-diagnosis of breast cancer 15 years after end of Malmö Mammographic Screening Trial: follow-up study. BMJ 2006; 332:689-92.

45. Independent UK Panel on Breast Cancer Screening. The benefits and harms of breast cancer screening: an independent review. Lancet 2012; 380:1778-86.

46. Miller AB, Wall C, Baines CJ, Sun P, To T, Narod SA. Twenty five year follow-up for breast cancer incidence and mortality of the Canadian National Breast Screening Study: randomised screening trial. BMJ 2014; 348:g366.

47. JørgensenKJ, Gøtzsche PC. Overdiagnosis in publicly organised mammography screening programmes: systematic review of incidence trends. BMJ 2009; 339:b2587.

48. Elmore JG, Barton MB, Moceri VM, Polk S, Arena PJ, Fletcher SW. Ten-year risk of false positive screening mammograms and clinical breast examinations. N Engl J Med 1998; 338:1089-96.

49. Welch HG, Frankel BA. Likelihood that a woman with screen-detected breast cancer has had her "life saved" by that screening. Arch Intern Med 2011; 171:2043-6.

50. Gøtzsche PC, Olsen O. Is screening for breast cancer with mammography justifiable? Lancet 2000; 355:129-34.

51. Leung GM, Lam TH, Thach TQ, Hedley AJ. Will screening mammography in the East do more harm than good? Am J Public Health 2002; 92:1841-6.
52. Autier P, Boniol M, Middleton R, Doré JF, Héry C, Zheng $\mathrm{T}$, et al. Advanced breast cancer incidence following population-based mammographic screening. Ann Oncol 2011; 22:1726-35.

53. Jørgensen KJ, Zahl PH, Gøtzsche PC. Breast cancer mortality in organised mammography screening in Denmark: comparative study. BMJ 2010; 340:c1241.

54. Heleno B, Thomsen MF, Rodrigues DS, Jørgensen KJ, Brodersen J. Quantification of harms in cancer screening trials: literature review. BMJ 2013; 347:f5334.

55. Rasmussen K, Jørgensen KJ, Gøtzsche PC. Citations of scientific results and conflicts of interest: the case of mammography screening. Evid Based Med 2013; 8:83-9.

56. Hoffman RM, Lewis CL, Pignone MP, Couper MP, Barry MJ, Elmore JG, et al. Decision-making processes for breast, colorectal, and prostate cancer screening: the DECISIONS survey. Med Decis Making 2010; 30(5 Suppl):53S-64S.

57. Illich I. A expropriação da saúde: nêmesis da medicina. Rio de Janeiro: Nova Fronteira; 1975.

58. Federação Brasileira de Instituições Filantrópicas de Apoio à Saúde da Mama. Documento de posição da FEMAMA para o diagnóstico precoce do câncer de mama. http://www.femama.org.br/no vo/arquivos/0.163891001286463380.pdf (acessado em 18/Abr/2016).

59. Hospital de Câncer de Barretos. Outubro Rosa 2013 no Hospital de Câncer de Barretos. Comunicado online. http:/ /www.hcancerbarretos.com.br/ institucional/unidades/82-noticias-institucional/ 938-uma-cidade-rosa (acessado em 18/Abr/2016).

60. Sociedade Brasileira de Mastologia. Como lidar com os temores sobre o câncer de mama. Comunicado online. http://www.sbmastologia.com.br/ index.php?option=com_content\&view=article\&i d=903:como-lidar-com-os-temores-sobre-o-can cer-de-mama\&catid=115\&Itemid $=707$ (acessado em 18/Abr/2016).

61. National Health Service. NHS breast screening helping you decide, 2015. https://www.gov.uk/ government/uploads/system/uploads/attach ment_data/file/440798/nhsbsp.pdf (acessado em 18/Abr/2016).

62. Jørgensen KJ. Is the tide turning against breast screening? Breast Cancer Res 2012; 14:107.

63. Pace LE, Keating NL. A systematic assessment of benefits and risks to guide breast cancer screening decisions. JAMA 2014; 311:1327-35.

64. World Health Organization. WHO position paper on mammography screening. http://apps.who.int/ iris/bitstream/10665/137339/1/9789241507936 eng.pdf?ua=1\&ua=1 (acessado em 18/Abr/2016).

65 . Heath I. It is not wrong to say no. BMJ 2009; 338:b2529. 


\section{Abstract}

The aim of this article was to discuss the recommendation of mammogram screening for breast cancer and its technical basis. The first part discusses criteria for the decision, which should be consistent with highquality scientific evidence. The second part discusses over-diagnosis (the greatest harm of screening) and its meaning in questioning the natural history of disease model. The third part summarizes studies on the efficacy, effectiveness, and harms of screening, showing that the latter (especially over-diagnosis and false-positives) are significant, shedding doubt on the balance between harms and benefits. In conclusion, the recommendation of mammogram screening at any age should be reconsidered by Brazilian health authorities.

Mass Screening; Breast Neoplasm; Secondary

Prevention

\section{Resumen}

El objetivo de este artículo fue discutir la recomendación del rastreo mamográfico del cáncer de mama y sus bases técnicas. La primera parte abarca consideraciones sobre criterios de decisión, que deben ser coherentes con evidencias científicas de alta calidad. La segunda parte discute sobre el diagnóstico, el mayor daño del rastreo, y su significado como cuestionador del modelo de la historia natural de la enfermedad. La tercera parte sintetiza estudios sobre eficacia, efectividad y daños del rastreo, mostrando que los últimos (principalmente sobre diagnósticos y falsos-positivos) son significativos y hacen que el equilibrio entre daños y beneficios sea dudoso. Se concluye que la recomendación de rastreo mamográfico debe ser reconsiderada por las autoridades sanitarias brasileñas en cualquier edad.

Tamizaje Masivo; Neoplasias de la Mama; Prevención Secundaria
Recebido em 21/Jan/2014

Versão final reapresentada em 23/Jun/2015 Aprovado em 09/Set/2015 